\title{
Inactivation of the NHEJ Activity of DNA-PKes Prevents Fanconi Anemia Pre-Leukemic HSC Expansion
}

\author{
Srinivas Chatla ${ }^{1}$, Andrew F. Wilson ${ }^{1}$, Qishen Pang ${ }^{1,2}$ \\ ${ }^{I}$ Division of Experimental Hematology and Cancer Biology, Cincinnati Children's Hospital Medical Center, Cincinnati, OH, USA \\ ${ }^{2}$ Department of Pediatrics, University of Cincinnati College of Medicine, Cincinnati, OH, USA
}

Fanconi anemia $(\mathrm{FA})$ is a genetic disorder characterized by bone marrow failure and high risk of cancer particularly leukemia. Here we show that inactivation of the non-homologous end-joining (NHEJ) activity of DNA-PKcs prevented DNA damage-induced expansion of FA pre-leukemic hematopoietic stem cells (HSCs). Furthermore, we performed serial BM transplantation to demonstrate that the DNA damage-induced expanded FA HSC compartment contained pre-leukemic stem cells that required the NHEJ activity of DNA-PKcs to induce leukemia in the secondary recipients. These results suggest that NHEJ may collaborate with FA deficiency to promote DNA damage-induced expansion of pre-leukemic HSCs.

Keywords: DNA damage, Fanconi anemia, Leukemic stem cells, Non-homologous end-joining

\section{Introduction}

Fanconi Anemia (FA) is a genetic disorder associated with bone marrow failure and high risk of cancer particularly leukemia (1-3). The disease is caused by defects in any of at least 22 genes $(F A N C A-W)$, which together constitute the so-called FA pathway engaged in some of the most important cellular processes such as DNA replication, cell-cycle control, and DNA damage response and repair (4-6). In the context of hematologic malignancies, FA commonly progresses from bone marrow (BM) failure

Received: June 5, 2019, Revised: August 5, 2019,

Accepted: August 15, 2019, Published online: August 31, 2019

Correspondence to Qishen Pang

Division of Experimental Hematology and Cancer Biology, Cincinnati Children's Hospital Medical Center, 3333 Burnet Avenue, Cincinnati, OH 45229, USA

Tel: +1-513-636-1152, Fax: +1-513-636-3768

E-mail: Qishen.pang@cchmc.org

(a) This is an open-access article distributed under the terms of the Creative Commons Attribution Non-Commercial License (http://creativecommons.org/ licenses/by-nc/4.0/), which permits unrestricted non-commercial use, distribution, and reproduction in any medium, provided the original work is properly cited.

Copyright (c) 2019 by the Korean Society for Stem Cell Research to a pre-leukemic myelodysplastic syndrome (MDS) stage and finally evolves to acute myeloid leukemia (AML). Patients with FA commonly develop pancytopenia during the first few years of life, and have high susceptibility of developing MDS or AML $(7,8)$. Currently, hematopoietic stem cell (HSC) transplantation is considered the best treatment for BM failure and leukemia in FA patients (9, 10).

It is postulated that the FA pathway promotes the error-free homologous recombination (HR) repair pathway while suppressing the error-prone non-homologous endjoining (NHEJ) pathway (11-14). In supporting this notion, recent studies have shown that FA deficiency enhanced the error-prone NHEJ repair, leading to increased genomic instability, and that genetic or pharmacological inhibition of the NHEJ pathway could rescue the FA phenotype $(11,13)$. However, the biological consequence on the interplay between FA pathway and the NHEJ pathway is not clear. In this study, we show that the NHEJ activity of DNA-PKcs is required for DNA damage-induced expansion of $\mathrm{Fanca}^{-/-}$pre-leukemic HSCs. 


\section{Materials and Methods}

\section{Mice and treatment}

$\mathrm{Fanca}^{-/-}$mice were generated by interbreeding the heterozygous Fanca ${ }^{+/-}$(C57BL/6:B6, CD45.2; Dr. Madeleine Carreau at Laval University). DNA-PKcs ${ }^{3 A / 3 A}$ mice $(\mathrm{C} 57 \mathrm{BL} / 6$ : B6, CD45.2; provided by Dr. Benjamin P. C. Chen at University of Texas Southwestern Medical Center) (15) were generated by interbreeding heterozygous $D N A-P K c s{ }^{+/ 3 A}$ mice. These mice were C57BL/6:B6, CD45.1 or 2 All the animals including BoyJ mice (C57BL/6:B6, CD45.1) were maintained in the animal barrier facility at Cincinnati Children's Hospital Medical Center. For in vivo mitomycin C (MMC; Sigma-Aldrich, St. Louis, MO) treatment, mice were intraperitoneal (i.p.) injected with $0.3 \mathrm{mg} / \mathrm{kg}$ of MMC weekly for consecutive 6 weeks (16). All animal experiments were performed in accordance with the institutional guidelines and approved by the Institutional Animal Care and Use Committee of Cincinnati Children's Hospital Medical Center (IACUC2018-0006).

\section{Isolation of bone marrow cells and flow cytometry analysis}

The femora and tibiae were harvested from the mice immediately after their sacrifice with $\mathrm{CO}_{2}$. Bone marrow (BM) cells were flushed from bones into Iscove's modified Dulbecco's medium (IMDM; Invitrogen) containing 10\% FCS, using a 21-guage needle and syringe. Low-density BM mononuclear cells (LDBMMNCs) were separated by Ficoll Hypaque density gradient (Sigma-Aldrich, St. Louis, MO) and washed with IMDM medium.

For flow analysis and cell sorting, the lineage marker (Lin) mixture (BD Biosciences, San Jose, CA) for BM cells from treated or untreated mice included the following biotinylated antibodies: CD3 $\varepsilon$ (145-2C11), CD11b (M1/70), CD45R/B220 (RA3-6B2), mouse erythroid cells Ly-76 (Ter119), Ly6G and Ly-6C (RB6-8C5). Other conjugated antibodies (BD sciences, San Jose, CA) used for surface staining included: CD45.1 (A20), CD45.2 (A104), Scal (D7), c-kit (2B8), CD48 (HM48-1), CD150 (9D1). For the cell sorting, lineage negative cells were enriched using lineage depletion reagents (StemCell Technologies) according to the manufacturer's instruction. The Lin-negative and LSK populations were acquired by using the FACSAria II sorter (BD Biosciences).

\section{Bone marrow transplantation (BMT)}

For determination of MMC-induced expansion of the Fanca $^{-1-}$ HSCs, 2,000 LSK cells, along with 200,000 c-Kit-depleted protector cells, were transplanted into le- thally irradiated BoyJ $\left(\mathrm{CD} 45.1^{+}\right)$recipients. The recipients were then i.p. injected with low dose of MMC $(0.3$ $\mathrm{mg} / \mathrm{kg}$ ) weekly for 6 weeks. Two days after the final injection, donor-derived (CD45.2 ${ }^{+}$) BM LSK cells were gated for analysis of the SLAM (LSKCD150 ${ }^{+} \mathrm{CD} 48^{-}$) cell population. For serial BMT, 2 million BM cells from the primary recipient mice were transplanted into sublethally irradiated secondary CD45. $1^{+}$recipient mice. Survival of the recipients was monitored and plotted by the KaplanMeier curve method.

\section{Cell-cycle and apoptosis analysis}

To analyze the cell cycle status of the HSC subsets, bone marrow cells were initially stained with antibodies against Lin $^{+}$cells, C-KIT, SCA-1, CD150 and CD48 described above. After incubation with these cell surface antibodies, the cells underwent fixation and permeabilization with transcription factor buffer set (BD Biosciences, \#562725) according to the manufacturer's instruction. After fixation, cells were incubated with APC-anti-Ki67 (BD Biosciences, \#558615), washed and stained with PI. Cells were analyzed by flow cytometry. For the apoptosis detection, bone marrow cells were stained with the antibodies for the HSC surface markers, and then stained with APC-Annexin V (BD Biosciences, \#550474) and 7 AAD. Annexin V-positive populations were determined as apoptotic cells using the FACS LSR II (BD Biosciences).

\section{Statistical analysis}

Student's $t$-test was performed using GraphPad Prism v8 (GrapPad software). Comparaison of more than 2 groups was analyzed by one-way Anova test. Values of $p<$ 0.05 were considered statistically significant. Results are presented as mean \pm SD. ${ }^{*}$ indicates $\mathrm{p}<0.05 ; * *=\mathrm{p}<0.01$.

\section{Results and Discussion}

We previously reported that chronic exposure of $\mathrm{Fanca}^{-/}$ mice to DNA crosslinking drug mitomycin $\mathrm{C}$ (MMC) induced the expansion of pre-leukemic HSCs (17). We have also shown that inactivation of Parpl in $\mathrm{Fanca}^{-/-}$HSPCs caused hyper-active NHEJ, which was required for the predisposition of $\mathrm{Fanca}^{-1-}$ HSPCs to leukemia in secondary recipients (18). To determine if pre-leukemic $\mathrm{Fanca}^{-/-}$ HSC expansion required the NHEJ pathway, we crossed the $\mathrm{Fanca}^{--}$mice with a strain carrying the knockin $D N A-P K c{ }^{3 A / 3 A}$ mutation, which selectively inactivates the NHEJ activity but does not affect the kinase activity of DNA-PKcs (15). To assess exclusively the response of HSCs without the effect of different BM microenviron- 
ment, we transplanted fetal LSK cells isolated from WT, $F_{a n c a^{-/-}}, D N A-P K c s^{3 A / 3 A}$ and DNA-PKcs ${ }^{3 A / 3 A}$ $\mathrm{Fanca}^{-/-}$embryos at E14.5 into lethally irradiated BoyJ mice. The recipients were exposed to DNA damage by intraperitoneally (ip) injection of a low dose of MMC (0.3 $\mathrm{mg} / \mathrm{kg} ; 15)$ weekly for 6 weeks. The recipient mice were sacrificed at the end of the treatment and analyzed for the frequency of donor-derived HSCs (CD45.2 ${ }^{+}$LSKCD150 ${ }^{+}$ CD48 ${ }^{-}$; SLAM). As shown in Fig. 1A, a significant expansion of HSCs was observed only in the recipients transplanted with the $\mathrm{Fanca}^{-/-}$LSK cells (Fig. 1A). In contrast, inactivation of the NHEJ activity of DNA-PKcs prevented MMC-induced expansion of the $\mathrm{Fanca}^{-/-}$
HSCs, as HSC expansion was not observed in recipients transplanted with $D N A-P K c{ }^{3 A / 3 A} \mathrm{Fanca}^{-1-}$ LSK cells (Fig. 1A). These results suggest that the NHEJ activity of $D N A-P K C s$ is required for DNA damage-induced expansion of $\mathrm{Fanca}^{-/-}$HSCs.

To determine if the abrogation of MMC-induced $\mathrm{Fanca}^{-/-}$ HSC expansion by NHEJ inactivation was associated with the status of apoptosis or quiescence, we analyzed apoptosis and cell cycle in the donor SLAM cell population. We found that MMC caused a significant increase in apoptosis in all three tested groups (DNA-PKCs ${ }^{3 A / 3 A}, \mathrm{Fanca}^{-/}$ and DNA-PKCs $\left.{ }^{3 A / 3 A} \mathrm{Fanca}^{-/-}\right)$of SLAM cells compared to the WT control (Fig. 1B), suggesting that the abrogation
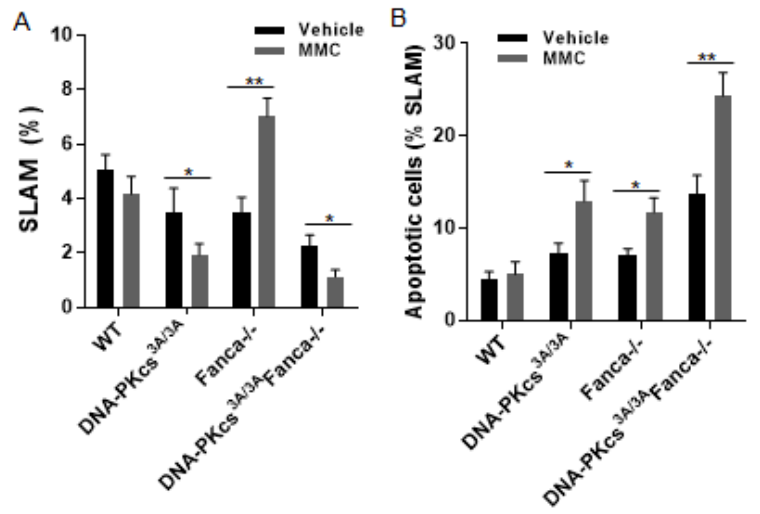

$E$

\begin{tabular}{|l|c|c|c|}
\hline & $\begin{array}{c}\mathrm{NE} \\
(\mathrm{K} / \mu \mathrm{l})\end{array}$ & $\begin{array}{c}\mathrm{RBC} \\
(\mathrm{M} / \mu \mathrm{l})\end{array}$ & $\begin{array}{c}\mathrm{Hb} \\
(\mathrm{g} / \mathrm{dl})\end{array}$ \\
\hline WT & $3.68 \pm 0.78$ & $10.5 \pm 1.69$ & $14.2 \pm 2.28$ \\
\hline DNA-PKcs & & & \\
\hline Fanca $^{-*}$ & $2.07 \pm 0.42^{\star}$ & $6.42 \pm 1.14^{\star}$ & $8.84 \pm 1.68^{\star}$ \\
\hline DNA-PKcs & $2.18 \pm 0.36^{\star}$ & $6.53 \pm 1.02^{\star}$ & $9.25 \pm 1.66^{\star}$ \\
\hline
\end{tabular}
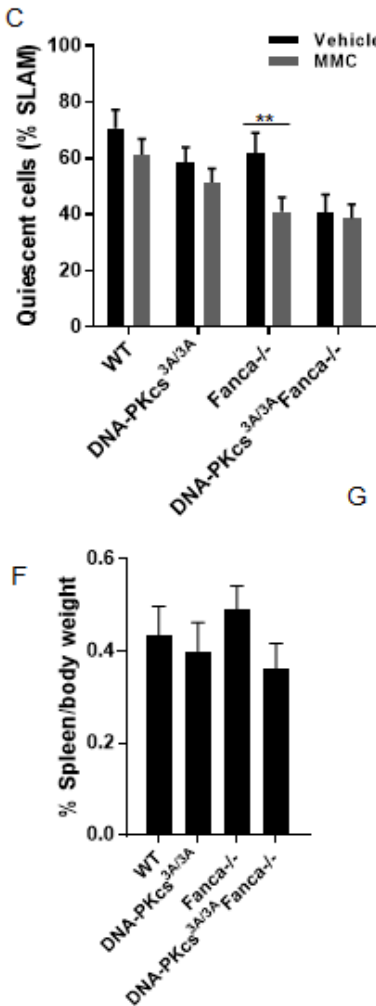

G
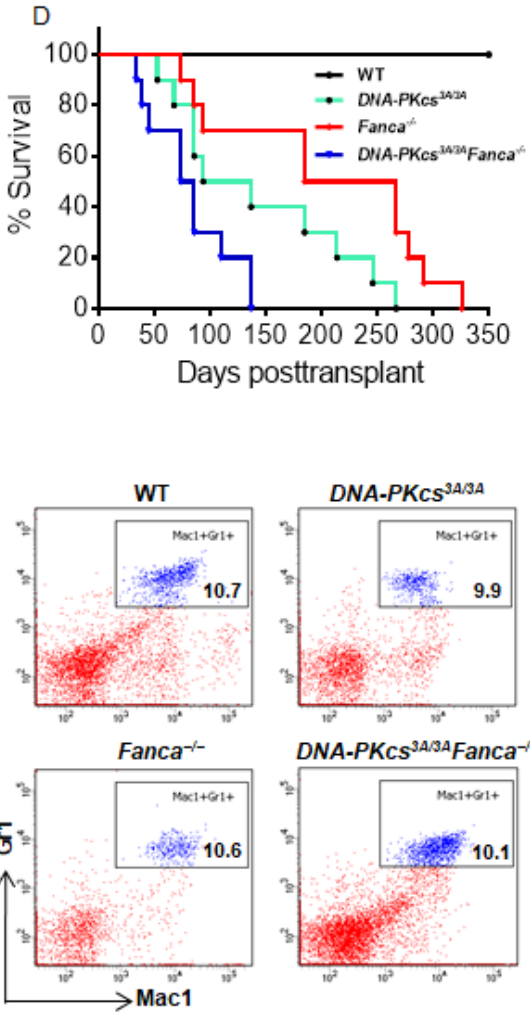

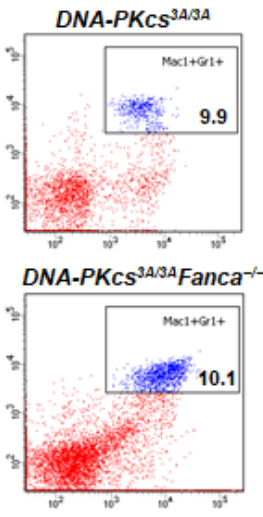

Fig. 1. Inactivation of the NHEJ activity of DNA-PKcs prevents the expansion of pre-leukemic Fanca ${ }^{-l-}$ HSCs. (A) Inactivation of the NHEJ activity of DNA-PKcs prevents MMC-induced expansion of the Fanca ${ }^{-1-}$ HSCs. 2,000 LSK cells from E14.5 embryos with the indicated genotype, along with 200,000 c-Kit-depleted protector cells, were transplanted into lethally irradiated BoyJ $\left(\mathrm{CD} 45.1^{+}\right.$) recipients. The recipients were i.p. injected with low dose of MMC $(0.3 \mathrm{mg} / \mathrm{kg})$ weekly for 6 weeks. Two days after the final injection, donor-derived (CD45.2 $\left.{ }^{+}\right)$ BM LSK cells were gated for analysis of the SLAM (LSKCD150 ${ }^{+} \mathrm{CD} 48^{-}$) cell population. (B) MMC induces a significant increase in apoptosis in DNA-PKCS ${ }^{3 A / 3 A}$, Fanca $^{-1-}$ and DNA-PKCS ${ }^{3 A / 3 A} \mathrm{Fanca}^{-1-}$ SLAM cells. Donor-derived (CD45.2 ${ }^{+}$) BM LSK cells were gated for SLAM population and analyzed for apoptosis by Annexin $\mathrm{V}$ and 7AAD. (C) MMC causes increased cell cycling in Fanca ${ }^{-1-}$ HSCs. Donor-derived $\left(C D 45.2^{+}\right)$BM LSK cells were gated for the SLAM population and analyzed for the cell cycle using Hochest 33342/Ki67 staining. (D) Survival of transplant recipients. 2,000 LSK cells from E14.5 embryos with the indicated genotype, along with 200,000 c-Kit-depleted protector cells, were transplanted into lethally irradiated Boyj recipients ( $n=10$ per group). MMC $(0.3 \mathrm{mg} / \mathrm{kg})$ was then administrated to the recipients weekly for 6 weeks. Survival of the recipients plotted by the Kaplan-Meier curve method and analyzed by the log-rank test. $(E \sim G)$ Anemia but no leukemia developed in primary recipient mice. The moribund mice described in (D) were subjected to analysis for peripheral blood count (E), and splenomegaly (F) and infiltration of mature myeloid cells (E) in the spleens. ${ }^{*} \mathrm{p}<0.05$; ${ }^{* *} \mathrm{p}<0.01$. 
of $\mathrm{Fanca}^{-1-}$ HSC expansion may be caused by increased apoptosis. However, MMC treatment induced significantly further reduction of quiescence in donor $\mathrm{Fanca}^{-/-}$SLAM cells but not in DNA-PKCs ${ }^{3 A / 3 A}$ or DNA-PKCs ${ }^{3 A / 3 A} \mathrm{Fanca}^{-/-}$ cells (Fig. 1C). Thus, it appears that increased cycling may play a role in MMC-induced expansion of $\mathrm{Fanca}^{-/-}$ HSCs.

To determine the biological consequence of DNA damage-induced $\mathrm{Fanca}^{-/-}$HSC expansion in the context of the NHEJ activity of DNA-PKcs, we performed serial BM transplantation and monitored the transplanted mice for leukemia development. The primary recipients transplanted with $D N A-P K c s^{3 A / 3 A} \mathrm{Fanca}^{-/-}$donor cells succumbed to BM failure in less than 150 days post-transplant (Fig. 1D). All primary recipients receiving $D N A-P \mathrm{Cs}^{3 A / 3 A}$ or $\mathrm{Fanca}^{-/-}$donor cells also died from BM failure, albeit less severe than those receiving $D N A$ $\mathrm{PKCs}^{3 A / 3 A} \mathrm{Fanca}^{-1-}$ donor cells (Fig. 1D). Analysis of the moribund mice showed peripheral neutropenia and anemia without signs of leukemia, as evidenced by the ab-

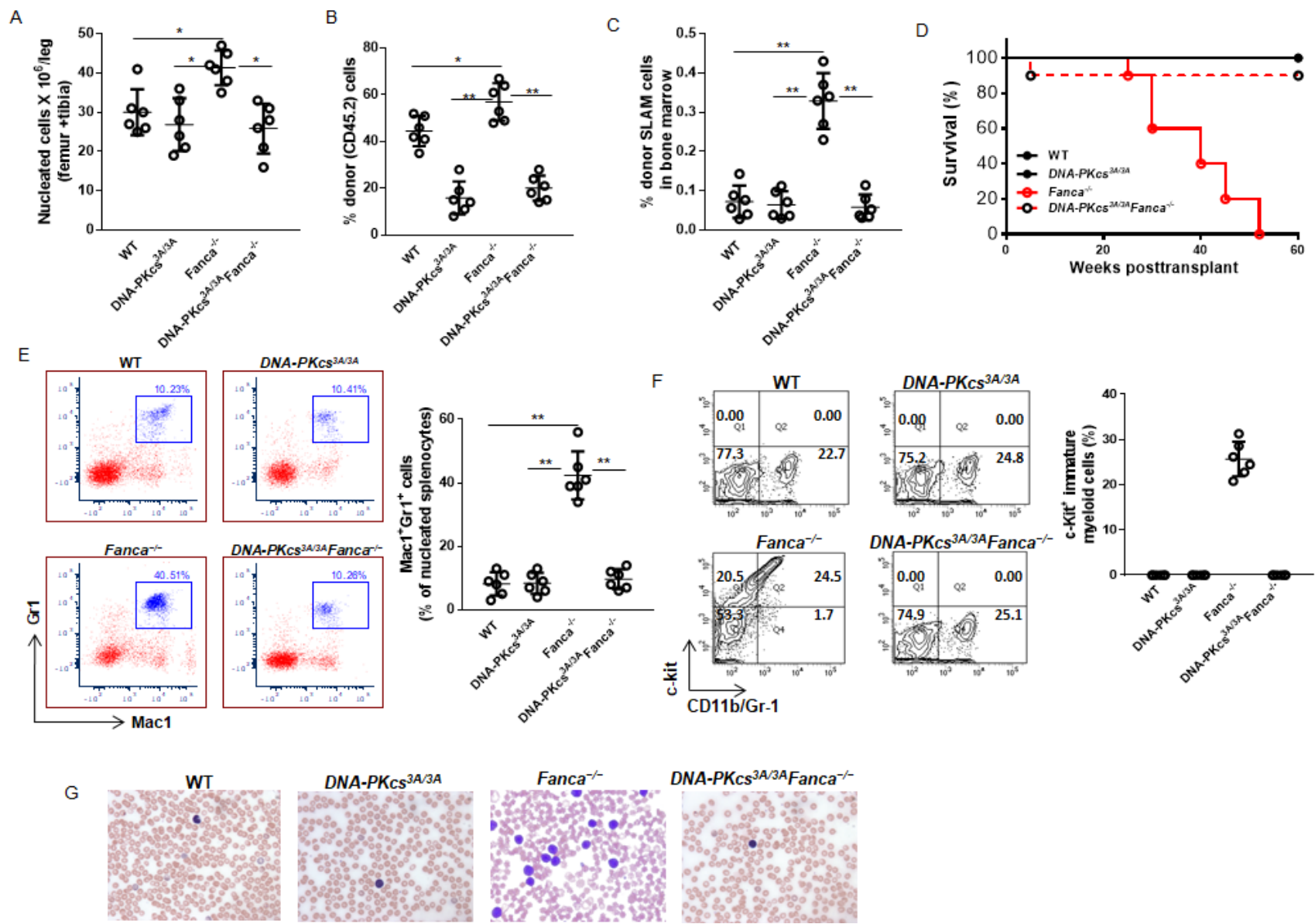

Fig. 2. The NHEJ activity of DNA-PKCS is required for the pre-leukemic Fanca ${ }^{-1-}$ HSCs to induce leukemia in the secondary recipients. $(\mathrm{A} \sim \mathrm{C})$ Inactivation of the NHEJ activity of DNA-PKcs restores normal levels of BM cellularity and phenotypic HSCs in the BM of the secondary recipients. 2,000 LSK cells from E14.5 embryos with the indicated genotype, along with 200,000 c-Kit-depleted protector cells, were transplanted into lethally irradiated BoyJ $\left(\mathrm{CD} 45.1^{+}\right)$recipients. The recipients were i.p. injected with low dose of $\mathrm{MMC}(0.3 \mathrm{mg} / \mathrm{kg})$ weekly for 6 weeks. Two days after the final injection, the mice were sacrificed and analyzed for BM cellularity (A), total donor (CD45.2) engraftment (B), and donor-derived SLAM (LSKCD150 ${ }^{+} \mathrm{CD} 48^{-}$) cells (C). (D) Fanca ${ }^{-1-}$ secondary recipients develop lethal leukemia. 2 million BM cells from the primary recipient mice described in (A) were transplanted into sublethally irradiated secondary $\mathrm{CD}_{45.1^{+}}$recipient mice. Survival of the recipients was monitored and plotted by the Kaplan-Meier curve method. $n=8 \sim 10$ mice for each group. (E, F) Increased myeloid infiltration in the spleen of the leukemic mice. Splenocytes from the recipient mice described in (D) were subjected to flow cytometry analysis for mature myeloid cells (E) and immature myeloid blasts (F). (G) Photomicrographs of Wright-Giemsa stain show increased blast cells in the peripheral blood smear from secondary recipient mice transplanted with Fanca ${ }^{-l-}$ donor cells. ${ }^{*} \mathrm{p}<0.05$; $* * p<0.01$. 
sence of splenomegaly and infiltration of mature myeloid cells in the spleens (Fig. $1 \mathrm{E} \sim \mathrm{G}$ ). However, we observed that recipients of $\mathrm{Fanca}^{-/-}$cells exhibited BM hypercellularity (Fig. 2A), and increased total donor-derived $\left(\mathrm{CD} 45.2^{+}\right)$cells (Fig. 2B) and accumulation of donor SLAM cells (Fig. 2C) in the BM, compared to those of WT, DNA-PKCs ${ }^{3 A / 3 A}$ or DNA-PKCs ${ }^{3 A / 3 A} \mathrm{Fanca}^{-1-}$ donor cells. Consistently, the secondary recipients of $\mathrm{Fanca}^{-1-}$ cohorts gave rise to lethal leukemias within 52 weeks (Fig. 2D). The $\mathrm{Fanca}^{-/-}$leukemic mice showed infiltration of mature myeloid cells (Fig. 2E) and immature myeloid blasts (Fig. 2F) in the spleen. Consistent with these, we observed increased population of immature myeloid blasts in the peripheral blood smear from secondary recipient mice transplanted with $\mathrm{Fanca}^{-/-}$donor cells (Fig. 2G). Thus, these results indicate that the expanded $\mathrm{Fanca}^{-/-}$ HSC compartment contains pre-leukemic stem cells that required the NHEJ activity of DNA-PKcs to induce leukemia in the secondary recipients.

In this report, we employed an in vivo system, in which we specifically inactivated the NHEJ activity of DNAPKcs in the $\mathrm{Fanca}^{-/-}$HSCs, to demonstrate that the NHEJ activity of DNA-PKcs is required for the expansion of pre-leukemic $\mathrm{Fanca}^{-/-}$HSCs. Our results suggest that the NHEJ pathway may functionally collaborate with FA deficiency in the process of leukemic transformation. We previously showed that loss of DNA damage response (DDR), due to FA deficiency, is correlated with the emergence and expansion of pre-leukemic $\mathrm{Fanca}^{-/-}$HSCs that give rise to leukemia in secondary transplanted recipients (17). However, how the multiple pathways (that is, FA, HR, NHEJ and other DDR pathways) cooperate within the DDR network in guarding against the initiation and progression of HSCs into leukemic transformation is not clear. Both FA and NHEJ pathways are involved in the repair of double-strand breaks (DSBs). Since the FA pathway can act to suppress the error-prone NHEJ pathway in DSB repair (11-14), it is conceivable that FA-deficient HSCs might preferentially use error-prone pathway for DSB repair, which may lead to high risk of pathological transformation. In this context, our finding is in line with the notion that impaired DDR due to FA deficiency and subsequently improper DSB repair as consequence of hyperactive NHEJ, resulting in genetic instability, may ultimately lead to leukemic transformation.

\section{Abbreviations:}

\begin{tabular}{ll}
\hline DDR & DNA damage response \\
FA & Fanconi anemia \\
HSC & hematopoietic stem cell \\
HR & homologous recombination \\
NHEJ & non-homologous end-joining \\
DSB & double-strand break \\
MMC & mitomycin C \\
BM & bone marrow \\
LDBMMNCs & low-density BM mononuclear cells \\
AML & acute myeloid leukemia \\
MDS & myelodysplastic syndrome \\
\hline
\end{tabular}

\section{Declarations}

\section{Acknowledgments}

We thank Dr. Madeleine Carreau (Laval University) for Fanca $^{+/-}$mice, and Dr. Benjamin P. C. Chen (University of Texas Southwestern Medical Center) for DNA-PKcs ${ }^{3 A / 3 A}$ mice. We also thank the Comprehensive Mouse and Cancer Core of the Cincinnati Children's Research Foundation (Cincinnati Children's Hospital Medical Center) for bone marrow transplantation service. This investigation was partially supported by NIH grants R01 HL076712, R01 HD089932. Q.P. was supported by a Leukemia and Lymphoma Scholar award.

\section{Potential Conflict of Interest}

The authors have no conflicting financial interest.

\section{Author Contributions}

S. C., designed research, performed research, analyzed data and wrote the paper; A. F. W., performed research; Q. P., designed research and wrote the paper.

\section{Availability of Data and Materials}

The datasets used and/or analyzed during the current study are available from the corresponding author on reasonable request.

\section{Consent for Publication}

Not applicable.

\section{Ethics Approval and Consent to Participate}

All animal procedures were approved by the Institutional Animal Care and Use Committee of Cincinnati Children's Hospital Medical Center prior to study initiation (IACUC protocol \# 2013-0159). 


\section{References}

1. Bagby GC Jr. Genetic basis of Fanconi anemia. Curr Opin Hematol 2003;10:68-76

2. Green AM, Kupfer GM. Fanconi anemia. Hematol Oncol Clin North Am 2009;23:193-214

3. Bogliolo M, Surrallés J. Fanconi anemia: a model disease for studies on human genetics and advanced therapeutics. Curr Opin Genet Dev 2015;33:32-40

4. Kottemann MC, Smogorzewska A. Fanconi anaemia and the repair of Watson and Crick DNA crosslinks. Nature 2013;493:356-363

5. Dong H, Nebert DW, Bruford EA, Thompson DC, Joenje $\mathrm{H}$, Vasiliou V. Update of the human and mouse Fanconi anemia genes. Hum Genomics 2015;9:32

6. Mamrak NE, Shimamura A, Howlett NG. Recent discoveries in the molecular pathogenesis of the inherited bone marrow failure syndrome Fanconi anemia. Blood Rev 2017; 31:93-99

7. Kutler DI, Singh B, Satagopan J, Batish SD, Berwick M, Giampietro PF, Hanenberg H, Auerbach AD. A 20-year perspective on the International Fanconi Anemia Registry (IFAR). Blood 2003;101:1249-1256

8. Vélez-Ruelas MA, Martínez-Jaramillo G, Arana-Trejo RM, Mayani H. Hematopoietic changes during progression from Fanconi anemia into acute myeloid leukemia: case report and brief review of the literature. Hematology 2006;11:331334

9. Mehta P, Locatelli F, Stary J, Smith FO. Bone marrow transplantation for inherited bone marrow failure syndromes. Pediatr Clin North Am 2010;57:147-170

10. Schneider $M$, Chandler $K$, Tischkowitz $M$, Meyer S. Fanconi anaemia: genetics, molecular biology, and cancer implications for clinical management in children and adults. Clin Genet 2015;88:13-24
11. Adamo A, Collis SJ, Adelman CA, Silva N, Horejsi Z, Ward JD, Martinez-Perez E, Boulton SJ, La Volpe A. Preventing nonhomologous end joining suppresses DNA repair defects of Fanconi anemia. Mol Cell 2010;39:25-35

12. Bunting SF, Nussenzweig A. Dangerous liaisons: Fanconi anemia and toxic nonhomologous end joining in DNA crosslink repair. Mol Cell 2010;39:164-166

13. Pace P, Mosedale G, Hodskinson MR, Rosado IV, Sivasubramaniam M, Patel KJ. Ku70 corrupts DNA repair in the absence of the Fanconi anemia pathway. Science 2010;329: 219-223

14. Bunting SF, Callén E, Wong N, Chen HT, Polato F, Gunn A, Bothmer A, Feldhahn N, Fernandez-Capetillo O, Cao L, $\mathrm{Xu} \mathrm{X}$, Deng CX, Finkel T, Nussenzweig M, Stark JM, Nussenzweig A. 53BP1 inhibits homologous recombination in Brcal-deficient cells by blocking resection of DNA breaks. Cell 2010;141:243-254

15. Zhang S, Yajima H, Huynh H, Zheng J, Callen E, Chen HT, Wong N, Bunting S, Lin YF, Li M, Lee KJ, Story M, Gapud E, Sleckman BP, Nussenzweig A, Zhang CC, Chen DJ, Chen BP. Congenital bone marrow failure in DNAPKcs mutant mice associated with deficiencies in DNA repair. J Cell Biol 2011;193:295-305

16. Carreau M, Gan OI, Liu L, Doedens M, McKerlie C, Dick $\mathrm{JE}$, Buchwald $\mathrm{M}$. Bone marrow failure in the Fanconi anemia group $\mathrm{C}$ mouse model after DNA damage. Blood 1998;91:2737-2744

17. Du W, Amarachintha S, Wilson A, Pang Q. The immune receptor Treml cooperates with diminished DNA damage response to induce preleukemic stem cell expansion. Leukemia 2017;31:423-433

18. Du W, Amarachintha S, Wilson AF, Pang Q. Hyper-active non-homologous end joining selects for synthetic lethality resistant and pathological Fanconi anemia hematopoietic stem and progenitor cells. Sci Rep 2016;6:22167 